\title{
Stochastic Spatial Models: From Simulations to Mean Field and Local Structure Approximations *
}

\author{
David Hiebeler \\ Center for Applied Math \\ 657 Rhodes Hall \\ Cornell University \\ hiebelerecam. cornell. edu
}

August 26, 1996

\begin{abstract}
A discrete stochastic spatial model for a single species is examined. First, detailed spatial simulations are performed using stochastic cellular automata. Then, several analytic approximations are made. First, two versions of mean field theory are presented: the infinite-dispersal mean field approximation, which is a metapopulation-like model, and the localdispersal mean field approximation, which incorporates the locality of the cellular automaton model but assumes that no spatial correlations develop in the lattice. Next, the local-dispersal mean field theory is generalized into several varieties of local structure theory, in which one assumes that groups of nearby sites in the lattice are correlated, and tracks such correlations under the action of the cellular automaton rule. Assuming such local correlations allows one to predict patch occupancy as well as the degree of clustering in the cellular automaton model much more accurately than mean field theory, especially in parameter regimes where mean field theory does poorly. Simulation and mean field theory are seen to be two opposite extremes of an entire spectrum of methods that may be used to investigate discrete spatial models.
\end{abstract}

\section{Introduction}

There has recently been growing interest in spatial issues in ecology, such as how locality of competition and range of dispersal play a role in ecosystem dynamics (Kareiva, 1994; McLaughlin and Roughgarden, 1993; Pacala, 1986a,b; Tilman, 1994). As a result, there is increasing interest among theoreticians in spatial models as well. These models are usually investigated via simulation (Colasanti and Grime, 1993; Darwen and Green, 1996; Etter and Caswell,

\footnotetext{
*Submitted to Journal of Theoretical Biology.
} 
1994; Inghe, 1989; McCarthy, 1996; Oborny, 1994; Silvertown et al., 1992), or else they are simplified by essentially throwing away all of the detailed spatial structure - giving the so-called metapopulation or mean-field models (Caswell and Cohen, 1991a,b, 1993; Hanski, 1991, 1994; Hanski and Gilpin, 1991; Hastings, 1991; Nee and May, 1992; Tilman, 1994). The latter type of model may be most appropriately termed "pseudo-spatial," since there is some notion of space involved, but no actual spatial structure. In some cases, both types of models are explored and compared, usually by constructing a mean-field approximation of a detailed spatial model (Caswell and Etter, 1993; Durrett and Levin, 1994a; McCauley et al., 1993; Rhodes and Anderson, 1996). This method of approximation involves the assumption in some form that "space doesn't matter." However, even this assumption may be made in more than one way. The most common method is to assume dispersal over arbitrarily long distances in the model, as in metapopulation models; this method is referred to as the infinite-dispersal mean field approximation here. The other method, called the local-dispersal mean field approximation, assumes that no spatial correlations develop in the lattice over time, but still takes into account the locality of the detailed spatial model. It is the latter method which will be generalized here.

Several researchers have recently been generalizing the mean-field approach in continuous-time models, yielding the so-called pair approximation method (Harada et al., 1995; Harada and Iwasa, 1994; Kubo et al., 1996; Levin and Durrett, 1996; Matsuda et al., 1992; Sato et al., 1994). Whereas continuous-time mean-field models involve only differential equations describing the frequencies of states of individual sites in the lattice, the pair approximation models also include equations for the frequencies of pairs of sites being in any given states. The pair approximation technique therefore incorporates some local spatial correlations.

Pair approximation apparently has not been applied to ecological models in discrete time, although these techniques have been applied to deterministic cellular automata in the physics literature (Gutowitz and Victor, 1987; Gutowitz et al., 1987; Schulman and Seiden, 1978; Wilbur et al., 1986), known variously as generalized mean field theory, cluster expansion, or local structure theory. Discrete-time models are often useful in ecology, for example when reproduction and mortality are strongly seasonal. The purpose of this study was to investigate the ability of local structure theory to predict a discrete-time stochastic spatial ecological model, under different assumptions about the patterns of spatial correlations among sites in the lattice.

\section{The Model}

We will use a version of the basic contact process (e.g., Durrett and Levin, 1994b) on a finite lattice in discrete time to model single-species dynamics. This model may also be thought of as a stochastic cellular automaton using the von Neumann neighborhood (i.e. using the four nearest neighbors, and not the diagonal neighbors). The sites in the lattice represent patches of space; thus this is a patch-occupancy model, and not an individual-based model. A site 
having value one represents the presence of the species at that site; a site in state zero represents an empty patch, or a patch whose population size is below some given threshold.

On each time step, the following things occur:

1. Colonization: each occupied site sends propagules to each of its four neighbors, independently with probability $\lambda$ each. That is, with probability $\lambda$ a propagule is sent to the "north" neighbor, with probability $\lambda$ a propagule is sent to the "east" neighbor, and so on. If a propagule lands on an occupied site, it is wasted and has no effect. If one or more propagules land on an empty site, then that site will be colonized, i.e. become occupied on the next time step. Thus, observe that an occupied site produces $X$ seeds on each time step, where $X$ has a binomial distribution with $n=4$ and probability $p=\lambda$.

2. Disturbance: Every site in the lattice is "disturbed" (becomes empty), independently with probability $\mu$. This includes sites that were just colonized; in other words, disturbance affects empty sites.

Each site in the lattice is therefore a nonlinear Markov chain, with the following transition probabilities:

$$
\begin{array}{ll}
P[0 \rightarrow 0]=1-\gamma(1-\mu) & P[1 \rightarrow 0]=\mu \\
P[0 \rightarrow 1]=\gamma(1-\mu) & P[1 \rightarrow 1]=1-\mu
\end{array}
$$

where $\gamma=1-(1-\lambda)^{n}$ is the colonization probability, and $n$ is the number of occupied sites among the four nearest neighbors. For the purposes of this study, $\lambda$ was held fixed with a value of 0.25 . Thus, on average an uncrowded site would produce one propagule (although depending on the value of $\mu$, new seeds may not have a very large chance of surviving even until the beginning of the next time step).

This detailed spatial model was first investigated via simulations; all simulations were performed on a $256 \times 256$ lattice with periodic boundary conditions. The simulations were begun with $50 \%$ of the sites in the lattice occupied, chosen at random. At each time step, $\rho_{1}$, the proportion of sites that were occupied (i.e. the patch occupancy probability), was measured. As in Caswell and Etter (1993), when the least-squares regression line through the values of $\rho_{1}$ for the last 100 time steps had a slope of less than 0.001 , the simulation was considered to be close enough to equilibrium, and stopped. At that time, the patch occupancy probability $\rho_{1}$ was averaged over the last 10 time steps, and that value recorded as the equilibrium patch occupancy probability for those parameter settings. Figure 1 shows the state of the lattice at the end of such a run, with $\mu=0.375$ and $\lambda=0.25$, and a random lattice with the same value of $\rho_{1}$ for comparison. The value of $\rho_{1}$ over time for that same simulation is shown in figure 2 . 

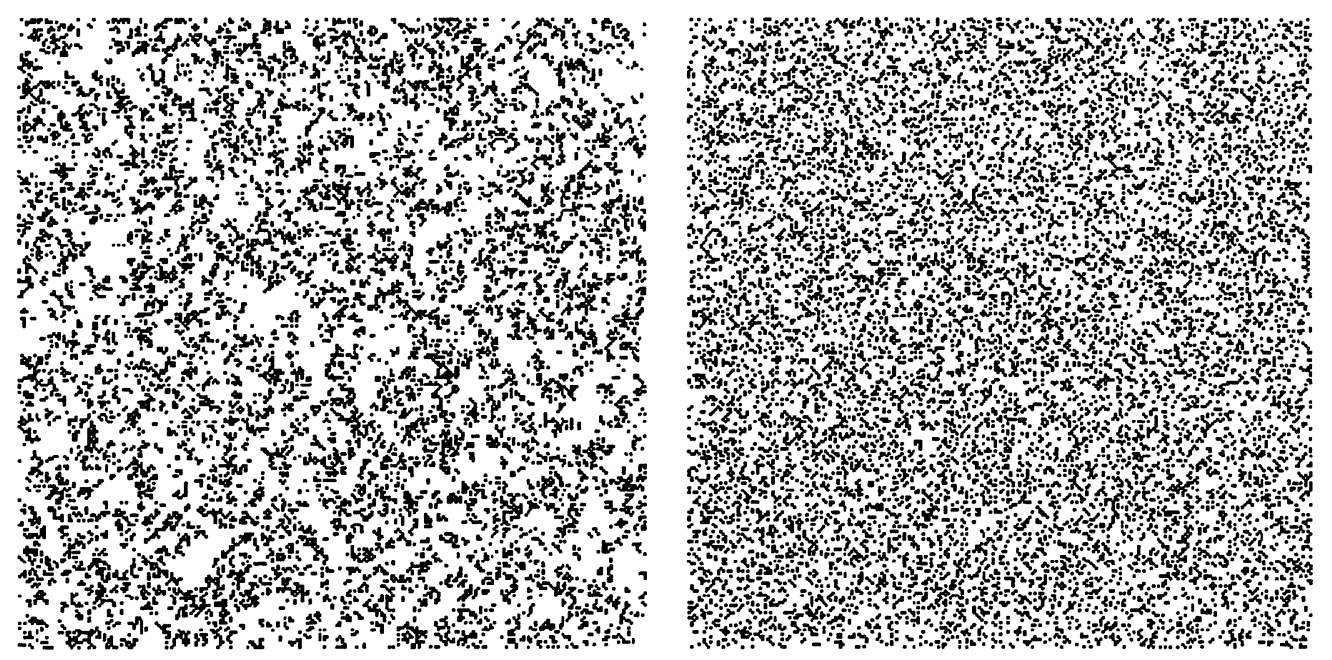

Figure 1: The left half of the figure shows the configuration of the lattice at the end of a run with $\mu=0.375, \lambda=0.25$. The measured patch occupancy probability in the lattice is roughly $\rho_{1}=0.19$. The right half of the figure shows a random lattice with the same density of occupied sites for comparison, making the clustering in the left figure more readily visible.

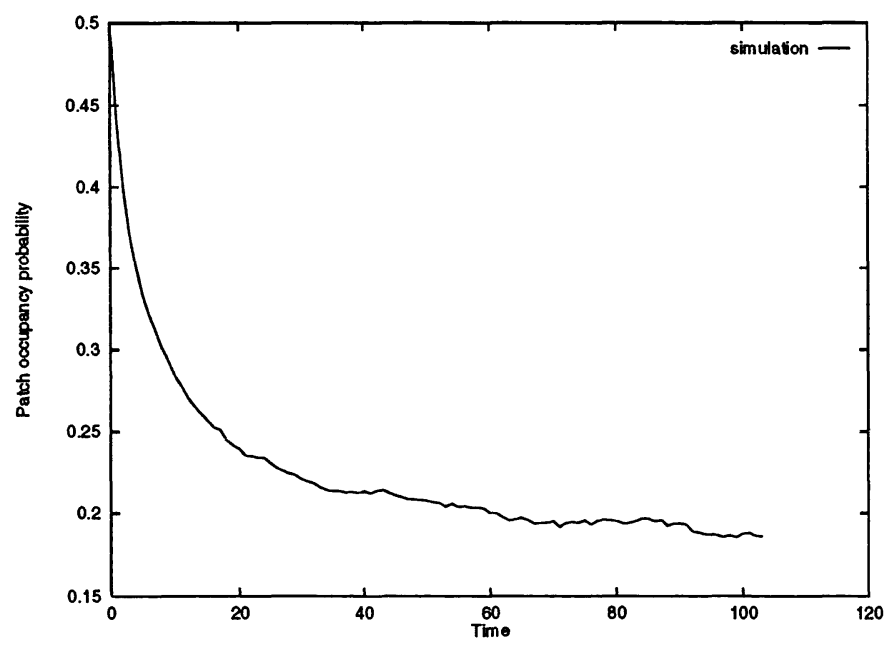

Figure 2: The graph displays the measured patch occupancy probability $\rho_{1}$ in the lattice over time, during the simulation on a $256 \times 256$ periodic lattice whose final configuration is shown in figure 1 . In the initial configuration of the lattice, $50 \%$ of the sites were occupied and $50 \%$ were empty, chosen at random. The parameter settings for this simulation were $\lambda=0.25$ and $\mu=0.375$. The simulation was stopped when the regression line had small enough slope. 


\section{The Infinite-Dispersal Mean Field Approxi- mation}

When constructing a non-spatial approximation of a spatial model, one wants to assume that "space doesn't matter." However, this assumption may be made in more than one way. For spatial models in discrete time, the most widely used technique is to assume infinite dispersal over the lattice (e.g., Caswell and Etter, 1993; Rhodes and Anderson, 1996). This is also the usual assumption in continuous-time mean field or metapopulation models as well (e.g., Durrett and Levin, 1994a; Tilman, 1994). In the model presented here, this means we assume that any given site still produces $X$ seeds, where $X$ has a binomial distribution as before with $n=4$ and $p=\lambda$; but rather than dropping the seeds on neighboring sites, instead the seeds will be dropped at random anywhere in the lattice. Thus, if there are $N$ sites in the lattice, there is probability $\frac{1}{N}$ that any given seed will land on some particular empty site being considered. If the patch occupancy probability is $\rho_{1}$ at the current time (also denoted by $P_{t}[1]$ when the time dependency needs to be made more explicit), then there will be $N \rho_{1}$ occupied sites; each one produces on average $4 \lambda$ seeds. Thus, the colonization probability for a given empty site is

$$
\gamma=1-\left(1-\frac{1}{N}\right)^{4 \lambda N \rho_{1}} \approx 1-e^{-4 \lambda \rho_{1}}
$$

where the approximation is very accurate when $N$ is reasonably large (on our $256 \times 256$ lattice $N=65,536$, which is more than adequate).

Recalling that in order for a site to change from state 0 (empty) at time $t$ to state 1 (occupied) at time $t+1$, that site must not only be colonized but also survive disturbance, it is easy to see that the expected proportion of occupied sites on the following time step, denoted by $P_{t+1}[1]$, is

$$
\begin{aligned}
P_{t+1}[1] & =P[0 \rightarrow 1] \cdot \rho_{0}+P[1 \rightarrow 1] \cdot \rho_{1} \\
& =\gamma(1-\mu) \rho_{0}+(1-\mu) \rho_{1},
\end{aligned}
$$

where $\gamma$ is as given in equation (1), $\rho_{0}=1-\rho_{1}$ is the proportion of sites which are empty at time $t, P[0 \rightarrow 1]$ is the probability that an empty site is colonized and survives disturbance, and $P[1 \rightarrow 1]$ is the probability that an occupied site is still occupied after the current time step, i.e. survives disturbance.

To find the equilibrium patch occupancy probability (i.e. the fixed point of this mapping), one needs to solve this equation for $P_{t+1}[1]=P_{t}[1]$. Since this is a transcendental equation, one must resort to numerical methods or simply iteration, but such methods are very simple and efficient. In this investigation, iteration was used to find the fixed point for this and the other approximation methods. 


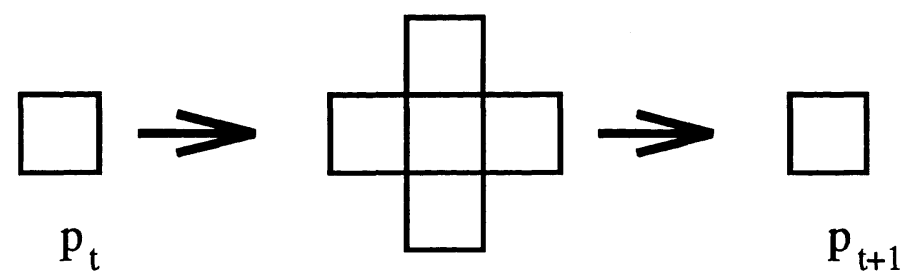

Figure 3: Under the assumptions of the local mean field theory (independence of sites in the lattice), the probabilities of single sites being empty or occupied are used to compute the probabilities of all pre-images in the center of the figure; the cellular automaton rule is then applied to the pre-images to compute the new probabilities for single sites on the following time step.

\section{The Local-Dispersal Mean Field Approxima- tion}

A second method of assuming that "space doesn't matter" is now presented; one which retains more of the flavor of the detailed cellular automaton rule. Instead of changing the behavior of seed dispersal, we still allow dispersal to occur only among the four nearest neighbors on the lattice. However, instead we assume that no spatial correlations are developing between the sites in the lattice as time goes on (an assumption which is obviously not true, of course). We assume that the probability of finding a particular site in state 1 , for example, is independent of the probabilities of any other site in the lattice being in state 0 or 1 . Under this assumption, we may characterize the state of the entire lattice at time $t$ with a single probability, $P_{t}[1]$, the probability that any given site is occupied (i.e. the value of $\rho_{1}$ at that time). Given this probability (and the complementary probability $P_{t}[0]=1-P_{t}[1]$, or $\left.\rho_{0}\right)$ and the assumption of the independence of sites, we may then calculate the probability that a group of five sites as shown in the center of figure 3 is in any particular configuration - the probability is simply the product of the probabilities of the sites within the group being in their respective states. This particular group of sites happens to be the preimage of a single site under the cellular automaton rule: that is, the state of the site in the center of the group at the next time step depends only upon the states of the sites in the pre-image at the previous time step, and not upon any other sites in the lattice. Thus we may compute the probability of finding a particular site in state 1 at time $t+1$ by conditioning on the state of the pre-image of that site at time $t$, and using the law of total probability. This may be written formally as:

$$
P_{t+1}[1]=\sum_{g \in \mathcal{G}} P_{t}[g] \cdot P[g \text { produces a site in state } 1],
$$

where $\mathcal{G}$ is the set of all pre-images of a single site (there are $2^{5}=32$ of them), and $P_{t}[g]$ is the probability of seeing the pre-image $g$ at time $t$. The last probability in equation (3) is simply the conditional probability that a site will be 
occupied at time $t+1$ given that the pre-image was in state $g$ at time $t$. Also, note that if we denote by $\#_{1}(g)$ the number of occupied sites in the pre-image $g$ and by $\#_{0}(g)$ the number of empty sites, then $P_{t}[g]=\rho_{0}^{\#_{0}(g)} \rho_{1}^{\#_{1}(g)}$ by the assumption of the independence of sites.

In fact, since under the cellular automaton rule used here, the probability of colonization depends only on how many of the four neighboring sites are occupied, and not their actual arrangement, we may rearrange the sum in equation (3), grouping together terms involving pre-images where the center site is occupied versus those where it is empty. One can finally obtain the following equation for the patch occupancy probability on the following time step under the local-dispersal mean field theory assumptions:

$$
P_{t+1}[1]=\gamma(1-\mu) \rho_{0}+(1-\mu) \rho_{1},
$$

where the colonization probability $\gamma$ is given by

$$
\gamma=\sum_{k=0}^{4}\left(\begin{array}{c}
4 \\
k
\end{array}\right) \rho_{1}^{k} \rho_{0}^{4-k}\left[1-(1-\lambda)^{k}\right] .
$$

This expression for the colonization probability $\gamma$ is obtained by conditioning on the number of occupied neighboring sites. Again, numerical methods or iteration can be used to find the fixed point of the mapping in equation (4), i.e. the equilibrium probabilities $\rho_{0}$ and $\rho_{1}$.

One can see that equations (2) and (4) are identical, the only difference being the different values of the colonization probabilities $\gamma$ given in equations (1) and (5). The colonization probabilities $\gamma$ for the two models are not identical, although the first-order terms do agree:

$$
\text { Infinite-dispersal: } \begin{aligned}
\gamma & =1-e^{-4 \lambda \rho_{1}} \\
& =4 \lambda \rho_{1}-8 \lambda^{2} \rho_{1}^{2}+\ldots \\
\text { Local-dispersal: } \quad \gamma & =\sum_{k=0}^{4}\left(\begin{array}{c}
4 \\
k
\end{array}\right) \rho_{1}^{k} \rho_{0}^{4-k}\left[1-(1-\lambda)^{k}\right] \\
& =4 \lambda \rho_{1}-12 \lambda \rho_{1}^{2}+6 \lambda^{2} \rho_{1}^{2}+\ldots
\end{aligned}
$$

The main difference between the infinite-dispersal and the local-dispersal mean field approximations is that the infinite-dispersal approximation allows for the possibility of "mass events" - for example, under this approximation, it is possible (although unlikely) for 10 sites to simultaneously drop a seed onto the same site. This cannot happen in the detailed spatial (cellular automaton) model, and also cannot happen in the local-dispersal mean field approximation - a site can have at most four seeds dropped on it at any given time, by its four neighbors. Although this distinction is fairly unimportant for this particular model, it may play a larger role in other models. Thus the choice of which nonspatial approximation to use for other models should be given some consideration. Finally, note that henceforth in this report, any mention of mean field theory refers to the local-dispersal mean field theory, and not the infinitedispersal approximation. 


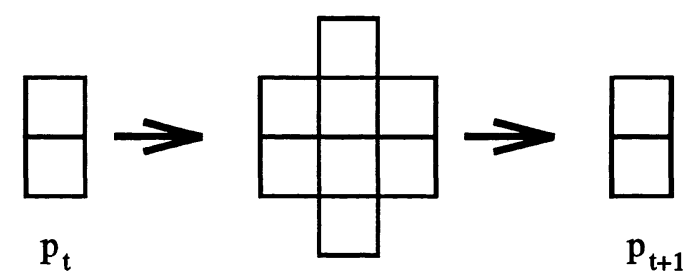

Figure 4: Under the assumptions of the local structure theory, adjacent sites in the lattice are correlated and non-adjacent sites are independent. The probabilities of all four possible $2 \times 1$ blocks are used to compute the probabilities of all pre-images in the center of the figure; the cellular automaton rule is then applied to the pre-images to compute the new probabilities for the $2 \times 1$ blocks on the following time step.

\section{Local Structure Theory}

The next step in approximating the detailed spatial model is a simple generalization of the local-dispersal mean field theory. Instead of assuming that all sites in the lattice are uncorrelated, we now assume that adjacent sites in the lattice are correlated, but that non-adjacent sites are independent. Furthermore, instead of characterizing the lattice by the probabilities of any given site being in state 0 or 1 , we now instead use the probabilities that any given $2 \times 1$ block of sites is in any of its four possible state configurations. Note that we will also assume rotational symmetry, so the probabilities will be the same for $1 \times 2$ blocks, and for notational convenience we will freely interchange $2 \times 1$ (vertical) and $1 \times 2$ (horizontal) blocks since they have the same probabilities. The methodology in equation (3) may then be generalized to apply to the probability $P_{t+1}\left[\begin{array}{l}a \\ b\end{array}\right]$ that any $2 \times 1$ block has one site in state $a$ and the other in state $b$ at time $t+1$ - we again condition on the state of the pre-image of a $2 \times 1$ block, and use the law of total probability:

$$
P_{t+1}\left[\begin{array}{l}
a \\
b
\end{array}\right]=\sum_{g \in \mathcal{G}} P[g] \cdot P\left\{g \text { produces the block }\left[\begin{array}{l}
a \\
b
\end{array}\right]\right\} .
$$

Figure 4 illustrates the process, and is the local structure theory analogue of figure 3 from the mean field approximation.

The main difficulty with the local structure theory is determining how to compute the probabilities of the pre-images shown in the center of figure 4, using the assumptions given. Gutowitz et al. (1987) discuss the problem for one-dimensional cellular automata, while Gutowitz and Victor (1987) suggest a generalization of the method to two dimensions. The problem is basically how to use the knowledge of the probabilities of $2 \times 1$ blocks of sites to compute the probabilities of larger blocks, for example $3 \times 1$ blocks. First, if we assume that our $2 \times 1$ block probabilities satisfy some basic consistency conditions (Gutowitz et al., 1987), then note that from the $2 \times 1$ block probabilities, we may compute 
the probabilities $\rho_{1}$ and $\rho_{0}$ of single sites being occupied and empty, by summing over appropriate $2 \times 1$ probabilities. For example $\rho_{1}=P[1]$ may be computed by summing over all $2 \times 1$ blocks with a 1 in the leftmost position:

$$
\rho_{1}=P[1]=P[10]+P[11] .
$$

Note that the consistency conditions mentioned above basically state that the same result is obtained if one sums over the probabilities of all $2 \times 1$ blocks with a 1 in the rightmost position. Also, the same idea allows one to compute $\rho_{0}=P[0]=P[00]+P[01]$. Next, we wish to compute the probability of a given $3 \times 1$ block " $a b c$ ", where $a, b$, and $c$ each have values 0 or 1 . First observe that the conditional probability that the rightmost site has value " $c$ " given that the leftmost two sites are in states " $a b$ ", written as $P[* * c \mid a b *]$ (where the * is a placeholder indicating that we don't care about the state of a particular site), under our local structure theory assumptions is equal to the probability $P[* * c \mid * b *]$, because the site in the third position is independent of the site in the first position. Thus,

$$
P[* * c \mid a b *]=P[* * c \mid * b *] .
$$

Then, noting that $P[* * c \cap a b *]=P[a b c]$, and $P[* * c \cap * b *]=P[* b c]$, we use the definition of conditional probability and equation (8) to write:

$$
\frac{P[a b c]}{P[a b *]}=\frac{P[* b c]}{P[* b *]}
$$

Now, the probability $P[a b *]$ is simply the probability $P[a b]$ which we know, and we can also compute $P[* b *]=P[b]$ by summing over $2 \times 1$ block probabilities as in equation (7). Thus, rearranging equation (9) yields the probability we want:

$$
P[a b c]=\frac{P[a b] P[b c]}{P[b]},
$$

and note that if $P[b]=0$, then by convention we shall set $P[a b c]=0$ as well.

The basic idea in the above is that in order to compute the probability of a larger block of sites, we cover it by overlapping small blocks whose probabilities multiply, and then divide out the probability of the overlapping region. This method may be applied to other shapes, such as any given pre-image as shown in figure 4 , by covering the pre-image with overlapping $2 \times 1$ "dominoes". Note however that there is more than one such tiling. For example, to compute the probability of a $2 \times 2$ block $P\left[\begin{array}{l}a b \\ c d\end{array}\right]$ using the $2 \times 1$ block probabilities, there are four possible ways: we could either cover the block with the tiles $\left[\begin{array}{l}a \\ c\end{array}\right]$, $\left[\begin{array}{ll}c & d\end{array}\right]$, and $\left[\begin{array}{l}b \\ d\end{array}\right]$, or we could use the tiles $\left[\begin{array}{ll}a & b\end{array}\right],\left[\begin{array}{l}b \\ d\end{array}\right]$, and $\left[\begin{array}{cc}c & d\end{array}\right]$, and so on. Different tilings can in fact give different probabilities. Although the lack of a unique way of extending probabilities may seem somewhat troubling, one may 


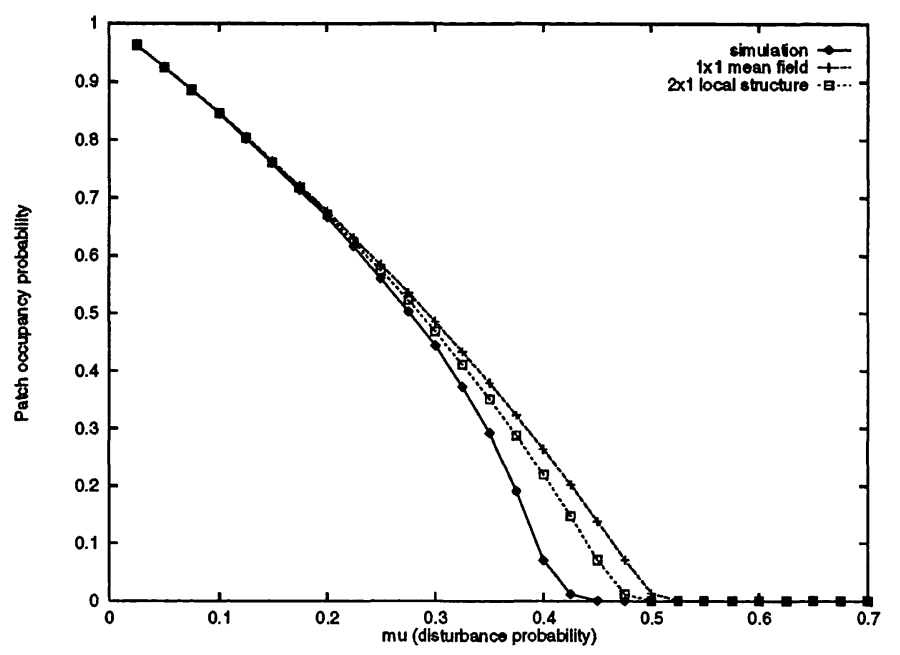

Figure 5: The graph displays the measured patch occupancy probability from the cellular automaton simulations (averaged over the last 10 time steps from each run after equilibrium was reached, as discussed in section 2), and as predicted by the mean field theory and $2 \times 1$ local structure theory approximations. This probability is shown for values of $\mu$ ranging from 0.025 to 0.7 in increments of 0.025 , with $\lambda$ held fixed at 0.25 .

simply think of them as different estimators for the larger probabilities, and choose one (or several) of them to try. The extension method used to compute the probabilities of pre-images in figure 4 for this study was

$$
P\left[\begin{array}{c}
a \\
b \text { cd } \\
e f f g \\
h
\end{array}\right]=\frac{P\left[\begin{array}{l}
a \\
c
\end{array}\right] P\left[\begin{array}{l}
b \\
c
\end{array}\right]}{P[c]} \frac{P\left[\begin{array}{l}
c \\
d
\end{array}\right]}{P[c]} \frac{P\left[\begin{array}{l}
b \\
e
\end{array}\right]}{P[b]} \frac{P\left[\begin{array}{l}
e \\
f
\end{array}\right]}{P[e]} \frac{P\left[\begin{array}{l}
f \\
g
\end{array}\right]}{P[f]} \frac{P\left[\begin{array}{l}
f \\
h
\end{array}\right]}{P[f]}
$$

where again the probability on the left hand side is set to zero if any of the denominators on the right vanish. Other tiling methods were tried, with similar results. Using the above formula to compute the probability of each pre-image $g$, equation (6) may then be used to compute the probabilities of the four $2 \times 1$ blocks on the next time step. One can find the fixed point as before, numerically or by iteration. Once the equilibrium probabilities of all four $2 \times 1$ blocks have been computed, the equilibrium patch-occupancy probability may be computed using equation (7).

The predicted patch-occupancy probabilities from the local-dispersal mean field approximation and the $2 \times 1$ local structure approximation are shown in figure 5 , along with the measured values from the cellular automaton simulations. The results from the infinite-dispersal mean field approximation are not shown, since they are so similar to the local-dispersal approximation predictions for this model. To more clearly compare the results of the two methods shown 


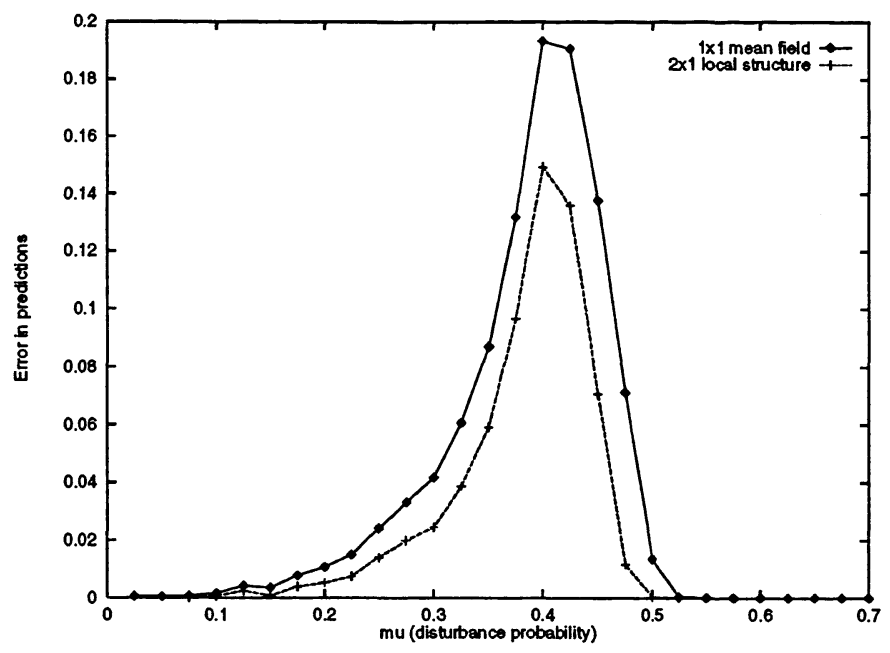

Figure 6: The error in the predicted patch occupancy probabilities is shown, i.e. the difference between the predicted values and the values measured from the simulations, for the mean field theory and $2 \times 1$ local structure theory approximations.

in figure 5 , figure 6 displays the error in the predictions, i.e. the difference between the predicted patch occupancy probabilities and the values measured from the actual simulations. As can be seen in figure 6, the largest error in the $2 \times 1$ predictions $(0.149$, when $\mu=0.400)$ is about 0.77 times as large as the maximum error in the mean field predictions (0.193). Thus, using $2 \times 1$ blocks to track some of the local spatial correlations in the approximations does improve the predictions. Note that both methods of approximation overestimate the patch occupancy probability $\rho_{1}$, quite drastically for intermediate values of $\mu$. This is most likely because the assumptions of independence of most sites in the lattice by the two approximations causes both methods to underestimate the degree of clustering in the actual simulations. When there is more clustering, i.e. when occupied sites tend to be near other occupied sites, more seeds are "wasted" by being dropped on neighboring occupied sites (and thus $\rho_{1}$ drops) than would be if nearby sites were not so highly correlated.

\section{More Local Structure}

The same basic ideas behind the $2 \times 1$ local structure theory approximation may be extended further: instead of using $2 \times 1$ correlation templates, other sizes and shapes may be used. For example, $4 \times 1$ and $2 \times 2$ templates were also investigated for this model. The idea behind equation (6) still applies, only now we use larger blocks which in turn have larger pre-images. The pre-images of $4 \times 1$ and $2 \times 2$ blocks are shown in figure 7 . To compute the probabilities of 

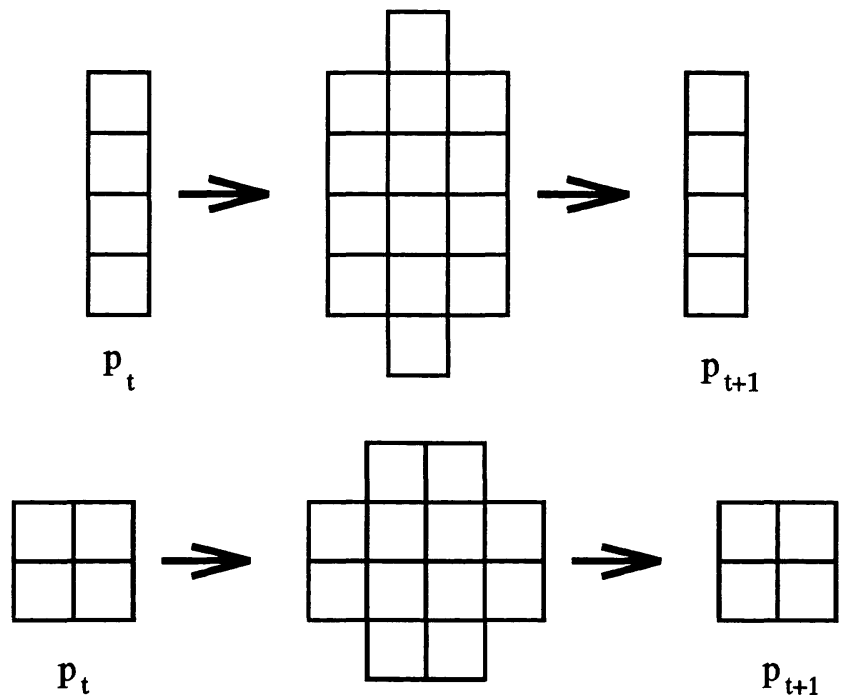

Figure 7: Larger correlation templates such as $4 \times 1$ (top) and $2 \times 2$ (bottom) may be used with the local structure theory. As before, the probabilities of all pre-images must then be computed, and the cellular automaton applied to the pre-images in order to compute the new block probabilities on the following time step.

pre-images needed in the general versions of equation (6), we again must cover the pre-images with overlapping blocks of sites whose probabilities we already know. We do this in a similar manner as before, summing over probabilities of known blocks in order to compute the probabilities of smaller blocks which we need for the tiling. The single-site patch occupancy probability $\rho_{1}$ is also computed from the block probabilities by summing over the appropriate set; for example to compute $\rho_{1}$ from the $4 \times 1$ block probabilities, one can sum the probabilities of all blocks which have a 1 in the leftmost position:

$\rho_{1}=P[1000]+P[1100]+P[1010]+P[1110]+P[1001]+P[1101]+P[1011]+P[1111]$.

Again, there are multiple ways of covering the pre-images with overlapping tiles; in fact, as the pre-images become larger, in general there are many more ways to cover them. A thorough investigation of the performance of all tiling methods was not performed here; rather, for the $4 \times 1$ and $2 \times 2$ cases, a single tiling was selected which used primarily the largest blocks whose probabilities were known. One could in fact tile the pre-images for example using only $2 \times 1$ tiles, but this would defeat the purpose of the larger scale local structure approximation, by neglecting the extra correlation information contained in the larger block probabilities.

To illustrate the power of these methods, figure 8 shows the error in patch occupancy probabilities predicted by all of the methods discussed (except for the infinite-dispersal mean field approximation): local-dispersal mean field, $2 \times$ 


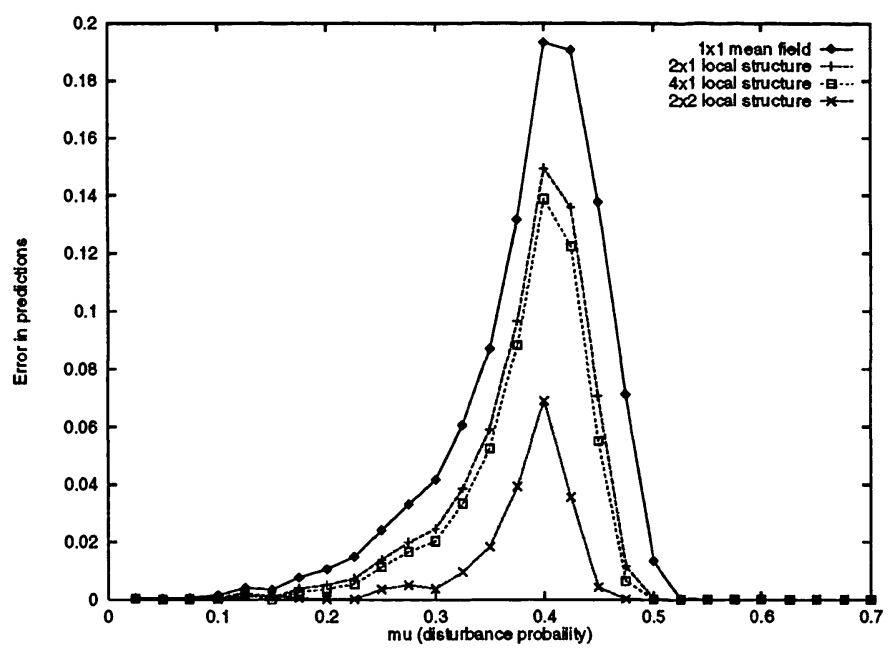

Figure 8: The error in the predicted patch occupancy probabilities is shown for the local-dispersal mean field, $2 \times 1,4 \times 1$, and $2 \times 2$ local structure approximations.

$1,4 \times 1$, and $2 \times 2$ local structure approximations. As can be seen in this graph, the maximum error in the $2 \times 2$ predictions $(0.069)$ is approximately 0.36 times as large as the maximum error in the mean field predictions (0.193), quite a significant reduction in error. It is also worth noting that $2 \times 2$ block probabilities do significantly better than $4 \times 1$ blocks. This suggests that if one is going to track spatial correlations among groups of four sites in the lattice, one is much better off using more detailed local correlations (square quadrats) as opposed to using correlations over a larger range of distances (longer transects). This is despite the fact that the $2 \times 2$ approximation actually requires fewer computations than the $4 \times 1$, since the $4 \times 1$ block has four times as many preimages $\left(2^{14}\right)$ as the $2 \times 2$ block $\left(2^{12}\right)$, because it has two sites more. In general, using larger blocks requires computing probabilities of more pre-images. If the blocks become too large, these computations become prohibitive in terms of both time and memory. The computations involving the largest block sizes used here run to completion on a Sparc-10 workstation within about one minute, and so are still quite feasible. As the blocks become larger, eventually they will reach the size of the entire lattice, at which time the approximation will in some sense become the simulation. In fact, this is a somewhat loose analogy, because the simulation is really only a single sample of the possible detailed behaviors of the system, while the local-structure computation involving blocks the size of the whole lattice will actually be computing the probabilities of all possible states of the entire lattice.

Following the example of Kubo et al. (1996), in addition to computing the patch occupancy probability, the conditional probability $q_{0 / 0}$ that a randomly chosen neighbor of an empty site is empty as well, was also computed. This 


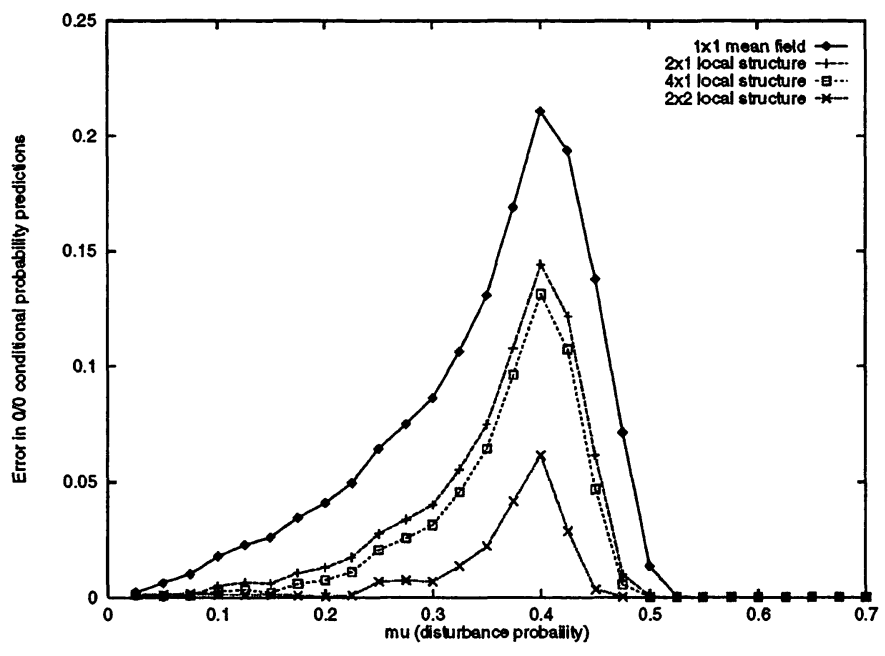

Figure 9: The error in the predicted $q_{0 / 0}$ conditional probabilities is shown for the local-dispersal mean field, $2 \times 1,4 \times 1$, and $2 \times 2$ local structure approximations.

is one measure of the degree of clustering in the lattice. If the sites in the lattice are not correlated, this probability will simply equal $\rho_{0}$; this is the value predicted by the mean field approximation. For the $2 \times 1$ local structure blocks, this conditional probability is simply $P[00] / \rho_{0}$. For the larger blocks, $q_{0 / 0}$ may be computed in a similar manner, by summing several of the block probabilities to compute $P[00]$, in a manner analogous to computing $\rho_{0}$ and $\rho_{1}$ from the larger block probabilities. The predictions of $q_{0 / 0}$ from the various methods is shown in figure 9 . For this value, the maximum error in the $2 \times 2$ approximation $(0.061$, again when $\mu=0.400$ ) was only 0.29 times as large as the maximum error in the mean field approximation (0.211). This difference is even more extreme than that in the predictions of the patch occupancy probabilities, which is not surprising given that the $q_{0 / 0}$ conditional probability reflects more of the local spatial structure in the lattice, and thus one would expect the methods which track local correlations to predict $q_{0 / 0}$ more accurately.

\section{Conclusions}

The two standard tools for investigating spatial models, simulation and infinitedispersal mean field theory, are seen to be extremes of an entire spectrum of methods. In addition, a second style of mean field involving only local dispersal exists, which is more open to generalization. Rather than keeping track of an entire lattice of spatial information, as in the simulations, or throwing away all of the spatial information, as in the mean field approximations, one may track local correlations of varying sizes and shapes using the local structure approxi- 
mation, a generalization of mean field theory. These techniques incorporate the local spatial effects of the cellular automaton rule on groups of nearby sites, in what is still essentially a mean-field-like approach. Predictions of the patch occupancy probability $\rho_{1}$ and clustering as measured by the conditional probability $q_{0 / 0}$ can be dramatically improved using even the very simplest version of the local structure approximation, $2 \times 1$ block probabilities, as has also been demonstrated in continuous-time models (e.g., Kubo et al., 1996). If one tracks correlations among larger groups of sites such as four-site blocks, the most accurate predictions are achieved when tracking detailed local correlations within $2 \times 2$ blocks of sites, as opposed to correlations across a larger scale of distances in $4 \times 1$ blocks. It is hoped that these techniques may help researchers to better understand the nature and effects of detailed local spatial interactions within discrete spatial models.

\section{Acknowledgements}

This work was conducted with financial support from NSF training grant BIR9113307, "The Dynamics of Heterogeneous Ecological and Evolutionary Systems," administered by Cornell University. The author would like to thank Howard Gutowitz for helpful discussions of these methods.

\section{References}

Caswell, H. \& Cohen, J. E. (1991a). Communities in patchy environments: A model of disturbance, competition, and heterogeneity. In: Ecological Heterogeneity (Kolasa, J. \& Pickett, S., eds) pp. 97-122. New York: SpringerVerlag.

Caswell, H. \& Cohen, J. E. (1991b). Disturbance, interspecific interaction, and diversity in metapopulations. Biol. J. Linn. Soc. 42,193-218.

CASwell, H. \& Cohen, J. E. (1993). Local and regional regulation of speciesarea relations: A patch-occupancy model. In: Species Diversity in Ecological Communities: Historical and Geographical Perspectives (Ricklefs, R. E. \& Schluter, D., eds), pp. 99-107. Chicago: The University of Chicago Press.

Caswell, H. \& EtTer, R. J. (1993). Ecological interactions in patchy environments: From patch occupancy models to cellular automata. In: Patch Dynamics (Powell, T., Levin, S. A., \& Steele, J., eds) pp. 93-109. New York: Springer-Verlag.

Colasanti, R. L. \& Grime, J. P. (1993). Resource dynamics and vegetation processes: A deterministic model using two-dimensional cellular automata. Func. Ecol. 7,169-176.

Darwen, P. \& Green, D. (1996). Viability of populations in a landscape. Ecol. Modelling 85,165-171. 
Durrett, R. \& Levin, S. A. (1994a). The importance of being discrete (and spatial). Theor. Pop. Biol. 46,363-394.

Durrett, R. \& Levin, S. A. (1994b). Stochastic spatial models: A user's guide to ecological applications. Phil. Trans. R. Soc. Lond. B343,329-350.

EtTer, R. J. \& CASwell, H. (1994). The advantages of dispersal in a patchy environment: Effects of disturbance in a cellular automaton model. In: Reproduction, Larval Biology and Recruitment in the Deep-sea Benthos, (Eckelbarger, K. \& Young, C., eds) pp. 284-305. New York: Columbia University Press.

Gutowitz, H. A. \& Victor, J. D. (1987). Local structure theory in more than one dimension. Complex Systems 1,57-68.

Gutowitz, H. A., Victor, J. D. \& Knight, B. W. (1987). Local structure theory for cellular automata. Physica D28,18-48.

HaNsKI, I. (1991). Single-species metapopulation dynamics: Concepts, models and observations. Biol. J. Linn. Soc. 42,17-38.

HANSKI, I. (1994). Patch-occupancy dynamics in fragmented landscapes. Tr. Ecol. Evo. 9,131-135.

Hanski, I. \& Gilpin, M. (1991). Metapopulation dynamics: Brief history and conceptual domain. Biol. J. Linn. Soc. 42,3-16.

Harada, Y., Ezoe, H., Iwasa, Y., Matsuda, H. \& Sato, K. (1995). Population persistence and spatially limited social interaction. Theor. Pop. Biol. 48,65-91.

Harada, Y. \& IwASA, Y. (1994). Lattice population dynamics for plants with dispersing seeds and vegetative propagation. Res. Pop. Ecol. 36,237-249.

Hastings, A. (1991). Structured models of metapopulation dynamics. Biol. J. Linn. Soc. 42,57-71.

INGHE, O. (1989). Genet and ramet survivorship under different mortality regimes - a cellular automata model. Jour. Theor. Biol. 138,257-270.

Kareiva, P. (1994). Space: The final frontier for ecological theory. Ecology $\mathbf{7 5}, 1$.

Kubo, T., Iwasa, Y. \& Furumoto, N. (1996). Forest spatial dynamics with gap expansion: Total gap area and gap size distribution. Jour. Theor. Biol. 180,229-246.

Levin, S. A. \& Durrett, R. (1996). From individuals to epidemics. In preparation. 
Matsuda, H., Ogita, N., Sasaki, A. \& Sato, K. (1992). Statistical mechanics of population. Prog. Theor. Phys. 88,1035-1049.

MCCARThy, M. A. (1996). Extinction dynamics of the helmeted honeyeater: Effects of demography, stochasticity, inbreeding, and spatial structure. Ecol. Modelling 85,151-163.

McCauley, E., Wilson, W. G. \& De Roos, A. M. (1993). Dynamics of agestructured and spatially structured predator-prey interactions: Individualbased models and population-level formulations. Am. Nat. 142,412-442.

Mclaughlin, J. F. \& Roughgarden, J. (1993). Species interactions in space. In: Species Diversity in Ecological Communities: Historical and Geographical Perspectives (Ricklefs, R. E. \& Schluter, D., eds), pp. 89-98. Chicago: University of Chicago Press.

NeE, S. \& MaY, R. (1992). Dynamics of metapopulations: Habitat destruction and competitive coexistence. J. Anim. Ecol. 61,37-40.

OвоRNy, B. (1994). Growth rules in clonal plants and environmental predictability - a simulation study. J. Ecol. 82,341-351.

PaCAla, S. W. (1986a). Neighborhood models of plant population dynamics: 2. multispecies models of annuals. Theor. Pop. Biol. 29,262-292.

PaCAla, S. W. (1986b). Neighborhood models of plant population dynamics: 4. single-species and multispecies models of annuals with dormant seeds. Am. Nat. 128,859-878.

Rhodes, C. \& Anderson, R. (1996). Persistence and dynamics in lattice models of epidemic spread. Jour. Theor. Biol. 180,125-133.

Sato, K., Matsuda, H. \& Sasaki, A. (1994). Pathogen invasion and host extinction in lattice structured populations. J. Math. Biol. 32,251-268.

Schulman, L. \& Seiden, P. (1978). Statistical mechanics of a dynamical system based on Conway's game of life. J. Stat. Phys. 19,293-314.

Silvertown, J., Holtier, S., Johnson, J. \& Dale, P. (1992). Cellular automaton models of interspecific competition for space - the effect of pattern on process. J. Ecol. 80,527-534.

Tilman, D. (1994). Competition and biodiversity in spatially structured habitats. Ecology 75,2-16.

Wilbur, W. J., Lipman, D. J. \& Shamma, S. A. (1986). On the prediction of local patterns in cellular automata. Physica D19,397-410. 